


\section{Guía Estadounidense para el Cumplimiento de Requerimientos para Acero Laminado Plano}

Lisa M. Benson Karen Reczek

Esta publicación se encuentra disponible de forma gratuita en: https://doi.org/10.6028/NIST.IR.8117es 



\title{
Guía Estadounidense para el Cumplimiento de Requerimientos para Acero Laminado Plano
}

\author{
Lisa M. Benson* \\ Karen Reczek \\ Coordinación de Normas
}

* Dakota Consulting

Esta publicación se encuentra disponible de forma gratuita en:

https://doi.org/10.6028/NIST.IR.8117es

Diciembre de 2013

Revisado en octubre de 2015

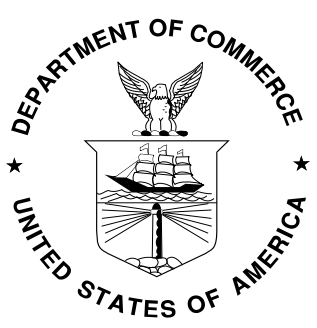

Departamento de Comercio de los Estados Unidos

Wilbur L. Ross, Jr., Secretaria de Comercio

Instituto Nacional de Normas y Tecnología Walter Copan, Director y Subsecretario de Comercio de NIST para Estándares y Tecnología 


\section{Agradecimientos}

El autor principal de este documento es Lisa M. Benson, de Dakota Consulting, bajo contrato de la Oficina de Coordinación de Normas (SCO) del Instituto Nacional de Normas y Tecnología (NIST). La asesoría adicional, investigación inicial y revisión del documento fueron proporcionadas por el personal de la Oficina de Coordinación de Normas del NIST, incluyendo a Mary Donaldson y Karen Reczek y el Laboratorio de Ingeniería del NIST, incluyendo a Timothy Foecke.

\section{Disclaimer}

This document was translated courtesy of the National Institute of Standards and Technology (NIST). The translation of this document was made by Ingesonic Ltd. with support from the ANSI Standards Alliance program.

This translation is not an official US Government or NIST translation. The US Government does not make any representations as to the accuracy of the translation.

The official English language version of this publication is available free of charge from the National Institute of Standards and Technology (NIST): https://doi.org/10.6028/NIST.IR.8117

\section{Renuncia}

Este documento fue traducido por cortesía del Instituto Nacional de Normas y Tecnología (NIST). La traducción de este documento fue realizada por Ingesonic Ltd. con el apoyo del programa ANSI Standards Alliance.

Esta traducción no es una traducción oficial del Gobierno de los Estados Unidos ni del NIST. EI Gobierno de los Estados Unidos no hace ninguna declaración sobre la exactitud de la traducción.

La versión oficial en inglés de esta publicación está disponible gratuitamente en el Instituto Nacional de Estándares y Tecnología (NIST): https://doi.org/10.6028/NIST.IR.8117 


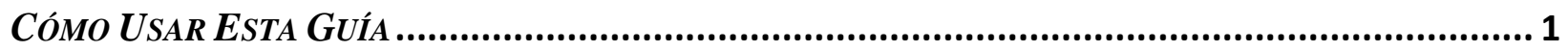

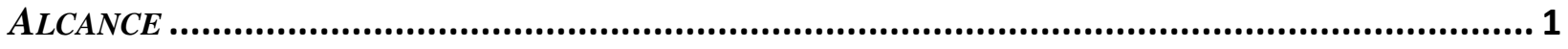

GENERALIDADES DEL MARCO NORMATIVO GENERAL DE LOS ESTADOS UNIDOS .......................... 1

AUTORIDADES REGULADORAS FEDERALES Y REGLAMENTOS TÉCNICOS (OBLIGATORIO) .............. 2

Oficina de Aduanas y Protección Fronteriza (CBP) ..................................................... 2

País de Origen: Marcado de Artículos Importados y Contenedores................................ 2

Requerimientos para la Obtención de Licencias ......................................................... 2

Porcentajes de Composición ............................................................................................ 3

Comisión Federal de Comercio (FTC) …………………................................................. 3

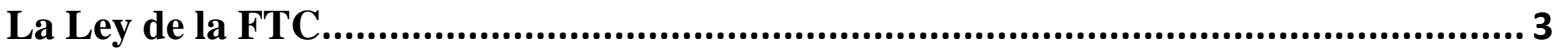

GENERALIDADES DE LOS MARCOS NORMATIVOS GENERALES DE LOS ESTADOS UNIDOS............... 3

AUTORIDADES REGULADORAS ESTATALES Y REGLAMENTOS TÉCNICOS (OBLIGATORIO)................ 4

Empaquetado y Etiquetado............................................................................................... 4

UPLR

Legislación sobre Tóxicos en Empaques....................................................................... 4

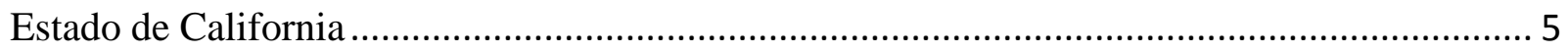

Plomo y Otras Substancias Tóxicas ............................................................................... 5

GENERALIDADES SOBRE EL MARCO DE NORMAS VOLUNTARIAS DE LOS ESTADOS UNIDOS............ 5

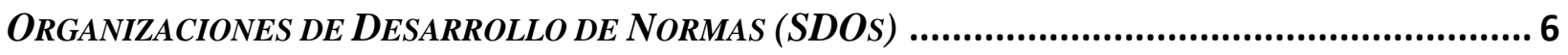

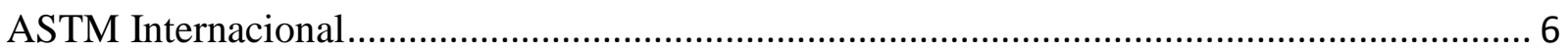

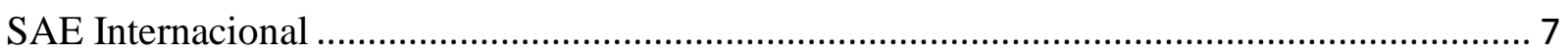

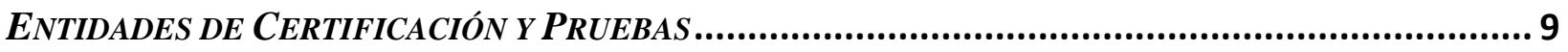

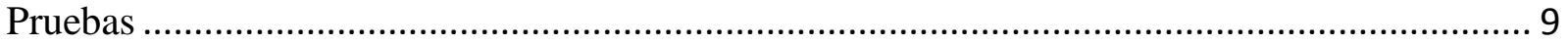

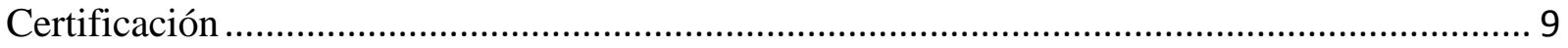

AGENCIAS DE GOBIERNO RELEVANTES DE ESTADOS UNIDOS ................................................. 9

Comisión Federal de Comercio..................................................................................... 9

Oficina de Aduanas y Protección Fronteriza (CBP) de los Estados Unidos ................................ 9

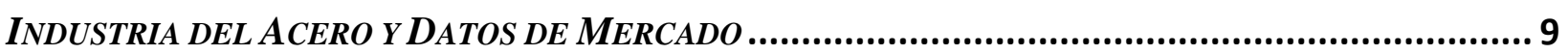

Asociaciones de Comercio de la Industria ............................................................................. 9

Instituto Americano del Hierro y el Acero (AISI) …...................................................... 9

Asociación de Fabricantes de Acero ............................................................................ 10

Datos de Mercado sobre el Acero Laminado ...................................................................... 10 



\section{Guía Estadounidense para \\ el Cumplimiento de Requerimientos para Acero Laminado Plano}

\section{Cómo UsAR Esta Guía}

- Los reglamentos son obligatorios

- Las normas son voluntarias (a menos que se encuentren "Incluidas por Referencia" en un reglamento)

- Los lineamientos pueden ser voluntarios (pero por lo general se trata de normas de hecho de la industria)

- Los textos en "Rojo" resaltan los requerimientos obligatorios

- Los textos en "Azul" indican un hipervínculo a un sitio web, página o documento en la web.

\section{ALCANCE}

La presente guía trata sobre acero laminado plano (en frio o en caliente), incluyendo el acero inoxidable, con aleación, sin aleación o hierro. No incluye productos terminados.

\section{Generalidades del Marco Normativo General de los Estados Unidos}

Una vez que el Congreso haya promulgado una ley, la agencia federal correspondiente (por ejemplo, la Comisión de Seguridad de Productos del Consumidor de Estados Unidos (CPSC), Comisión Federal de Comercio (FTC), Administración Nacional de Seguridad del Tráfico en las Carreteras (NHTSA), et al.) puede crear los reglamentos para implementar la ley. Antes de poder adoptar dichos reglamentos, la agencia federal correspondiente expedirá de manera ordinaria una notificación sobre la legislación propuesta (NPRM, siglas en inglés) para solicitar comentarios públicos en las reglas propuestas. Para brindar la oportunidad de comentario público, la respectiva agencia federal debe expedir proyectos de reglamentos o "Reglas Propuestas" que se publican en el Registro Federal como una notificación de Obstáculos Técnicos al Comercio de la Organización Mundial de Comercio (OTC OMC). La agencia revisa los comentarios y a continuación puede expedir una "Regla Final" que también es publicada en el Registro Federal, y posteriormente publicada de manera anual en el Código de Reglamentos Federales (CFR). En conjunto, las leyes de habilitación [publicadas en el Código de los Estados Unidos (USC) una vez aprobadas] y los reglamentos finales (publicados en el CFR) proporcionan un marco para la implementación y ejercicio de la mayoría de las leyes federales en los Estados Unidos. 
AUTORIDADES REGULADORAS FEDERALES Y REGLAMENTOS TÉCNICOS (OBLIGATORIO)

\begin{tabular}{|l|l|}
\hline Agencia & Alcance \\
\hline $\begin{array}{l}\text { Oficina de Aduanas y Protección Fronteriza } \\
\text { (CBP) }\end{array}$ & $\begin{array}{l}\text { País de Origen (para la mayoría de productos } \\
\text { importados, licienciamiento y composición) }\end{array}$ \\
\hline $\begin{array}{l}\text { Oficina de Aduanas y Protección Fronteriza } \\
\text { (CBP) }\end{array}$ & $\begin{array}{l}\text { Sistema de Monitoreo y Análisis para la } \\
\text { Importación de Acero }\end{array}$ \\
\hline $\begin{array}{l}\text { Oficina de Aduanas y Protección Fronteriza } \\
\text { (CBP) }\end{array}$ & Porcentajes de Composición \\
\hline Comisión Federal de Comercio (FTC) & Prácticas Desleales de Comercio \\
\hline
\end{tabular}

\section{Oficina de Aduanas y Protección Fronteriza (CBP)}

\section{País de Origen: Marcado de Artículos Importados y Contenedores \\ Título 19, Código de los Estados Unidos, Capítulo 4, Sección 1304 \\ Todos los productos importados a los Estados Unidos deben cumplir con los reglamentos de $\underline{19}$ CFR 134, Marcado del País de Origen. Estos reglamentos requieren que cada artículo de origen extranjero o (su contenedor) importado a los Estados Unidos se encuentre marcado en un lugar visible, de manera legible, indeleble y permanente en tanto lo permita la naturaleza del artículo (o contenedor), de esta manera se indica al comprador final en los Estados Unidos, el nombre en inglés del país de origen del artículo al momento de la importación.}

\section{Requerimientos para la Obtención de Licencias}

Todas las importaciones de los Estados Unidos de productos básicos de acería están sujetos a los requerimientos de licencia de importación según el 19 CFR 360 Sistema para el Monitoreo y Análisis para la Importación de Acero a través del sistema para el Monitoreo y Análisis para la Importación de Acero (SIMA) basado en la Internet. El propósito del sistema SIMA es proporcionar al público datos estadísticos sobre las importaciones de acero que ingresan a los Estados Unidos siete semanas antes de lo que normalmente estaría disponible para el público. Los datos agregados reunidos de las licencias se ponen a disposición del público de manera semanal siguiendo la revisión por parte del Departamento. Cualquier importador, empresa importadora, agente de aduana o agente del importador con una dirección en los Estados Unidos puede registrar y obtener el número de identificación del usuario necesario para iniciar sesión en el sistema automático de expedición de licencias para la importación de acero. Las empresas extranjeras pueden obtener un número de identificación de usuario si tienen una dirección en los Estados Unidos a través de la cual puedan ser contactados; no se aceptarán apartados postales.

El 15 febrero 2003, una Regla Final fue publicada en el registro Federal extendiendo el programa de licencias hasta marzo 21 de 2017. 


\section{Porcentajes de Composición}

El Reglamento de la Oficina de Aduanas y Protección Fronteriza (CBP) de EE.UU. 19 CFR 141.89 presenta información adicional que se requiere al momento de ingresar con hierro o acero clasificable en el capítulo 72 o bajo los títulos 7301 a 7307, de la Nomenclatura Arancelaria Armonizada de los Estados Unidos (HTSUS). Se requiere una declaración en la forma de un análisis de fresadora o certificado de pruebas de fresadora, indicando los porcentajes por peso o carbono y cualquier elemento metálico que contengan los artículos. Se requiere un certificado de pruebas de fresadora para cada ingreso de un producto de acero laminado plano sin aleación y con revestimiento incluido en el Capítulo 72 de la HTSUS.

\section{Para más detalles consulte en la CBP:}

Terminología y Métodos para Marcado del País de Origen sobre Importaciones de los Estados Unidos

Lo que Todo Miembro de la Comunidad Comercial Debe Saber acerca de: Acero Laminado Plano sin Aleación y con Revestimiento

\section{Comisión Federal de Comercio (FTC)}

\section{La Ley de la FTC}

Título 15, Código de los Estados Unidos, Capítulo 2, Sección 41-58

La Ley de la FTC prohíbe ampliamente los actos o prácticas injustas o fraudulentas dentro del comercio o aquellas que lo afecten. La Comisión encontrará fraude si, ya sea por la inclusión o exclusión información, probablemente:

- Se desvía a los clientes que actúan de manera razonable bajo las circunstancias, y

- Se afecta la elección del consumidor o su conducta, por consecuencia llevando a un perjuicio.

La Ley de la FTC le permitió a la FTC promulgar reglamentos con el propósito de prohibir actos o prácticas injustas o fraudulentas.

\section{GENERALIDADES DE LOS MARCOS NORMATIVOS GENERALES DE LOS ESTADOS UNIDOS}

Un creciente número de áreas están siendo amparadas tanto por estatutos federales como estatales, incluyendo la protección al consumidor, el empleo y el reglamento de alimentos y medicamentos. (Las leyes estatales dan cabida a las leyes federales más estrictas que tratan el mismo problema.) Cuando el gobernador del Estado firma una ley, esta se convierte en una ley estatal. Una vez que una ley ha sido sancionada por un Estado, es responsabilidad de la agencia estatal correspondiente el crear los reglamentos necesarios para implementar la ley. 


\section{AUTORIDADES REGULADORAS ESTATALES Y REGLAMENTOS TÉCNICOS (OBLIGATORIO)}

En los Estados Unidos, algunas leyes y reglamentos estatales que son sancionados resultan más rigurosos que las leyes federales. Estas leyes incluyen reglamentos para productos, etiquetas, empaques, restricciones químicas, etc.

\begin{tabular}{|l|l|}
\hline Agencia & Alcance \\
\hline $\begin{array}{l}\text { Autoridades Estatales Responsables de Pesos y } \\
\text { Medidas }\end{array}$ & Etiquetado \\
\hline $\begin{array}{l}\text { Centro de Información sobre Tóxicos en } \\
\text { Empaques (TPCH) }\end{array}$ & Empaquetado \\
\hline $\begin{array}{l}\text { Oficina de Evaluación de Peligros a la Salud } \\
\text { Ambiental de California (OEHHA) }\end{array}$ & Químicos Tóxicos \\
\hline
\end{tabular}

\section{Empaquetado y Etiquetado}

\section{UPLR}

Las Leyes y Reglamentos Uniformes en las áreas de Metrología Legal y Calidad de Combustibles para Motor (UPLR), Manual NIST 130, Reglamento de Empaquetado y Etiquetado Uniforme (UPLR), han sido adoptados en la ley de 45 de los 50 estados de Estados Unidos. El propósito de estos reglamentos es brindar información precisa y adecuada sobre la identidad y cantidad de los contenidos de paquetes para que los compradores puedan realizar comparaciones de precios y cantidades.

El UPLR requiere que el empaquetado que no es del consumidor lleve una etiqueta especificando la identidad del bien básico; el nombre y lugar de negocios del fabricante, empacador o distribuidor; y la cantidad neta de contenidos en términos de peso o medida de masa, o el conteo numérico en una ubicación uniforme en la parte exterior del paquete. Toda la información debe presentarse claramente el idioma inglés.

\section{Legislación sobre Tóxicos en Empaques}

Esta legislación fue originalmente propuesta por el Consejo para la Reducción de Fuentes (Source Reduction Council) de la Coalición de Gobernadores del Noreste (CONEG) en 1989. Se desarrolló en un esfuerzo para reducir la cantidad de metales pesados en el empaquetado y componentes de empaques que se venden o distribuyen a través de los Estados Unidos. La ley está diseñada para eliminar gradualmente el uso y presencia de mercurio, plomo, cadmio y cromo hexavalente del empaquetado. La legislación se ha adoptado con éxito en 19 estados.

\section{Para más detalles consulte la documentación técnica del Centro de Información sobre} Tóxicos en Empaques:

\section{$\underline{\text { Hoja de Datos sobre Tóxicos en Empaques }}$}




\title{
Estado de California
}

\section{Plomo y Otras Substancias Tóxicas}

California regula el plomo y muchas otras substancias y químicos a través de la Ley de Agua Potable Segura y Control de Sustancias Tóxicas de 1986, comúnmente conocida como Proposición 65 o Prop 65 (Código de Salud y Seguridad de California. Sección 25249.6, et seq.) Estos acuerdos proporcionan lineamientos para límites sugeridos. La Lista de Sustancias Peligrosas de la Proposición 65 se conserva y actualiza a medida que se identifican nuevos químicos.

El siguiente lenguaje de advertencia es requerido en los productos vendidos en California si éstos contienen químicos incluidos en la lista de la Proposición 65 y la cantidad de exposición causada por el producto no se encuentra definida dentro de los límites de seguridad:

ADVERTENCIA: Este producto contiene químicos conocidos por el Estado de California debidd a que causan cáncer y defectos de nacimiento u otros daños reproductivos.

\author{
Para más detalles sobre información oficial de California de la Proposición 65, \\ consulte: \\ Oficina de Evaluación de Peligros a la Salud Ambiental de California (OEHHA), \\ Proposición 65 en Lenguaje Claro, y \\ Noticias sobre la Prop65 de Novedades sobre la Proposición 65
}

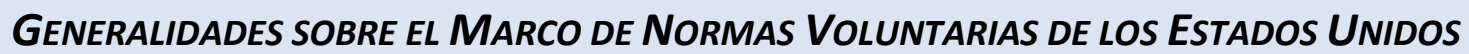

El sistema de Estados Unidos para el desarrollo de normas es impulsado por el sector privado. La mayoría de normas de Estados Unidos son voluntarias y se desarrollan a través de métodos de consenso que reflejan las necesidades de los productores y fabricantes, usuarios $y$ consumidores, y el gobierno. El Instituto Nacional Estadounidense de Estándares (ANSI) (una organización no gubernamental, sin ánimo de lucro) coordina las actividades del sector privado de la comunidad de desarrollo de normas voluntarias en los Estados Unidos. Existen cientos de normas voluntarias que desarrollan organizaciones en EE.UU responsables por la estandarización en muchas industrias diferentes y sectores comerciales. El Instituto Nacional de Normas y Tecnología (NIST), parte del Departamento de Comercio de los Estados Unidos, es el laboratorio nacional de metrología para los Estados Unidos. El NIST proporciona la infraestructura de medición técnica para apoyar el comercio internacional y el sistema de medición comercial. EI NIST, a través de su Oficina de Coordinación de Normas, proporcionar asesoría y coordina la participación Federal en el marco de las normas. 


\title{
ORGANIZACIONES DE DESARROLLO DE NORMAS (SDOS)
}

\section{ASTM Internacional}

\author{
100 Barr Harbor Drive \\ P.O. Box C700 \\ West Conshohocken, PA 19428-2959 USA \\ Teléfono: +1.610 .832 .9500$ \\ Directorio de Personal
}

ASTM Internacional (ASTM) desarrolla y preserva normas de consenso y métodos de prueba que pertenecen a los productos laminados.

El comité de ASTM responsable de los laminados es el Comité A01 - Acero, Acero Inoxidable y Aleaciones Relacionadas

Los siguientes comités de ASTM relevantes para los productos laminados incluyen:

\begin{tabular}{|l|l|}
\hline$\underline{\text { A01.06.03 }}$ & Métodos de Prueba y Prácticas Recomendadas \\
\hline$\underline{\text { A01.17 }}$ & Acero Inoxidable Laminado Plano y Forjado \\
\hline$\underline{\text { 001.19 }}$ & Lámina y Cinta de Acero \\
\hline
\end{tabular}

Los ejemplos de normas ASTM para productos laminados incluyen:

\begin{tabular}{|l|l|}
\hline $\begin{array}{l}\text { ASTM A } \\
\text { 6/A6M }\end{array}$ & $\begin{array}{l}\text { Especificación de Requerimientos Generales para Barras, Placas, } \\
\text { Formas y Tablestacado de Acero Estructural Laminado }\end{array}$ \\
\hline ASTM A & Especificación para Acero, Cinta, Carbono (Porcentaje Máximo de \\
109/A 109M & 0,25), Laminado en Frío \\
\hline $\begin{array}{l}\text { ASTM A } \\
\text { 480/A480M }\end{array}$ & $\begin{array}{l}\text { Especificación de Requerimientos Generales para Acero Placa, } \\
\text { Lámina y Cinta Laminado Plano y Resistente al Calor }\end{array}$ \\
\hline ASTM A 505 & $\begin{array}{l}\text { Especificación para Acero, Lámina y Cinta, Aleación, Laminado en } \\
\text { frío y Laminado en Caliente, Requerimientos Generales para }\end{array}$ \\
\hline ASTM A 506 & $\begin{array}{l}\text { Especificación para Acero, Lámina y Cinta en Aleación y Aleación } \\
\text { Estructural, Laminado en Caliente y Laminado en Frío }\end{array}$ \\
\hline ASTM A 507 & $\begin{array}{l}\text { Especificación para Extrusión de Acero con Aleación, Lámina y } \\
\text { Cinta, Laminado en Caliente y Laminado en Frío }\end{array}$ \\
\hline $\begin{array}{l}\text { ASTM A } \\
\text { 522/A522M }\end{array}$ & $\begin{array}{l}\text { Especificación para Bridas, Accesorios, Válvulas y Partes de Acero } \\
\text { con Aleaciones de Níquel al 8 y 9\% Forjados o Laminados para } \\
\text { Servicio a Baja Temperatura }\end{array}$ \\
\hline $\begin{array}{l}\text { ASTM A } \\
\text { 564/A564M }\end{array}$ & $\begin{array}{l}\text { Especificación para Barras y Formas de Acero Inoxidable con } \\
\text { Laminado en Caliente y Envejecimiento por Endurecimiento } \\
\text { Terminado en Frío }\end{array}$ \\
\hline ASTM A & Especificación para Acero, Lámina, Carbono, Estructural y Alta \\
\hline
\end{tabular}




\begin{tabular}{|l|l|}
\hline 568/A568M & $\begin{array}{l}\text { Resistencia, Baja Aleación, Laminado en Caliente y Laminado en } \\
\text { frío, Requerimientos Generales para }\end{array}$ \\
\hline $\begin{array}{l}\text { ASTM A } \\
\text { 578/A578M }\end{array}$ & $\begin{array}{l}\text { Especificación para Examen Ultrasónico con Haz Recto de Placas } \\
\text { de Acero Laminado para Aplicaciones Especiales }\end{array}$ \\
\hline ASTM A & $\begin{array}{l}\text { Especificación para Acero, Lámina y Cinta, Alta Resistencia, Baja } \\
\text { 606/A606M }\end{array}$ \\
& $\begin{array}{l}\text { Aleación, Laminado en Caliente y Laminado en frío, con Resistencia } \\
\text { Mejorada a la Corrosión Atmosférica }\end{array}$ \\
\hline
\end{tabular}

\section{SAE Internacional}

400 Commonwealth Drive

Warrendale, PA 15096-0001

Teléfono: +1.724 .776 .4841$

SAE Internacional es una asociación global con más de 128,000 ingenieros y expertos técnicos relacionados con las industrias aeroespacial, automotriz y de vehículos comerciales. Una de las competencias centrales de SAE Internacional es el desarrollo de normas de consenso voluntarias. Algunas de las normas relacionadas con laminado plano incluyen:

\begin{tabular}{|c|c|}
\hline SAE AMS 5501 & $\begin{array}{l}\text { Lámina, Cinta, y Chapa de Acero, Resistente a la Corrosión, } \\
\text { 19cr - 9.5ni (304), Laminado en Frío, Resistencia a la Tracción } \\
125 \text { Ksi ( } 862 \mathrm{Mpa})\end{array}$ \\
\hline SAE AMS 5517 & $\begin{array}{l}\text { Lámina y Cinta de Acero, Resistente a la Corrosión, Laminado } \\
\text { en Frío 18cr - 8ni (Sae 30301), Resistencia a la Tracción } 125 \text { Ksi } \\
\text { (862 Mpa) }\end{array}$ \\
\hline SAE AMS 5518 & $\begin{array}{l}\text { Lámina y Cinta de Acero, Resistente a la Corrosión, Laminado } \\
\text { en Frío } 18 \mathrm{cr}-8 \mathrm{ni} \text {, Resistencia a la Tracción } 150 \mathrm{Ksi} \text { (1034 Mpa) }\end{array}$ \\
\hline SAE AMS 5519 & $\begin{array}{l}\text { Lámina y Cinta de Acero, Resistente a la Corrosión, Laminado } \\
\text { en Frío 18cr - 8ni (Sae 30301), Resistencia a la Tracción } 185 \text { Ksi } \\
\text { (1276 Mpa) }\end{array}$ \\
\hline SAE AMS 5529 & $\begin{array}{l}\text { Lamina y Cinta de Acero, Resistente a la Corrosión, } 17 \mathrm{cr} \text { - } \\
7.1 \mathrm{ni} \text { - } 1.1 \text { al, Templado con Solución y Laminado en Frío, } \\
\text { Susceptible de Endurecimiento por Precipitación, Espesor } \\
\text { Nominal de } 0.0015 \text { a } 0.100 \text { Pulgadas (0.038 To } 2.54 \mathrm{Mm} \text { ) }\end{array}$ \\
\hline SAE AMS 5546 & $\begin{array}{l}\text { Lámina y Cinta de Acero, Resistencia a la Corrosión y } \\
\text { Resistencia al Calor Moderado } 16.5 \mathrm{cr}-4.5 \mathrm{ni}-2.9 \mathrm{mo}-0.10 \mathrm{n} \\
\text { Laminado en Frío, Templado }\end{array}$ \\
\hline SAE AMS 5810 & $\begin{array}{l}\text { Cable plano de acero, resistente al calor y la corrosión, } 15 \mathrm{cr} \text { - } \\
25.5 \mathrm{ni}-1.2 \mathrm{mo}-2.1 \mathrm{ti}-0.006 \mathrm{~b}-0.30 \mathrm{v}, 1800 \text { Grados F ( } 982 \\
\text { Grados C) Tratado por Solución, Estirado en Frío o Laminado } \\
\text { en Frío y Por Envejecimiento, Electrodo Consumible Fundido }\end{array}$ \\
\hline SAE AMS 5902 & $\begin{array}{l}\text { Lámina y Cinta de Acero, Resistente a la Corrosión, 18cr - 8ni } \\
\text { (Sae 30301), Laminado en Frío, Dureza 3/4, Resistencia a la }\end{array}$ \\
\hline
\end{tabular}




\begin{tabular}{|c|c|}
\hline & Tracción 175 Ksi (1207 Mpa) \\
\hline SAE AMS 5903 & $\begin{array}{l}\text { Lámina, Cinta y Placa de Acero, Resistente a la Corrosión, 18cr } \\
\text { - 9.0ni (Sae } 30302) \text {, Laminado en Frío, Dureza 1/4, Resistencia } \\
\text { a la Tracción } 125 \text { Ksi ( } 862 \mathrm{Mpa} \text { ) }\end{array}$ \\
\hline SAE AMS 5904 & $\begin{array}{l}\text { Lámina y Cinta de Acero, Resistente a la Corrosión, } 18 \mathrm{cr} \text { - } \\
\text { 9.0ni (Sae } 30302 \text { ), Laminado en Frío, Dureza 1/2, Resistencia a } \\
\text { la Tracción } 150 \text { Ksi (1034 Mpa) }\end{array}$ \\
\hline SAE AMS 5905 & $\begin{array}{l}\text { Lámina y Cinta de Acero, Resistente a la Corrosión, 18cr - } \\
\text { 9.0ni (Sae 30302), Laminado en Frío, Dureza 3/4, Resistencia a } \\
\text { la Tracción } 175 \text { Ksi (1207 Mpa) }\end{array}$ \\
\hline SAE AMS 5906 & $\begin{array}{l}\text { Lámina y Cinta de Acero, Resistente a la Corrosión, 18cr - } \\
\text { 9.0ni (Sae 30302), Laminado en Frío, Dureza Total, Resistencia } \\
\text { a la Tracción } 185 \text { Ksi (1276 Mpa) }\end{array}$ \\
\hline SAE AMS 5907 & $\begin{array}{l}\text { Lámina, Cinta y Placa de Acero, Resistente a la Corrosión y al } \\
\text { Calor, 17cr - 12ni - 2.5mo (Sae 30316), Laminado en Frío, } \\
\text { Dureza 1/4, Resistencia a la Tracción } 125 \text { Ksi (862 Mpa) }\end{array}$ \\
\hline SAE AMS 5910 & $\begin{array}{l}\text { Lámina, Cinta y Placa de Acero, Resistente a la Corrosión, 19cr } \\
\text { - 9.2ni (Sae } 30304) \text {, Laminado en Frío, Resistencia a la } \\
\text { Tracción } 125 \text { Ksi (862 Mpa) }\end{array}$ \\
\hline SAE AMS 5911 & $\begin{array}{l}\text { Lámina y Cinta de Acero, Resistente a la Corrosión, } 19 \mathrm{cr} \text { - } \\
\text { 9.2ni (Sae } 30304) \text {, Laminado en Frío, Dureza 1/2, Resistencia a } \\
\text { la Tracción } 150 \mathrm{Ksi} \text { (1034 Mpa) }\end{array}$ \\
\hline SAE AMS 5912 & $\begin{array}{l}\text { Lámina y Cinta de Acero, Resistente a la Corrosión, 19cr - } \\
\text { 9.2ni (Sae } 30304) \text {, Laminado en Frío, Dureza 3/4, Resistencia a } \\
\text { la Tracción } 175 \mathrm{Ksi} \text { (1207 Mpa) }\end{array}$ \\
\hline SAE AMS 5913 & $\begin{array}{l}\text { Lámina y Cinta de Acero, Resistente a la Corrosión, } 19 \mathrm{cr} \text { - } \\
\text { 9.2ni (Sae 30304), Laminado en Frío, Dureza Total, Resistencia } \\
\text { a la Tracción } 185 \text { Ksi (1276 Mpa) }\end{array}$ \\
\hline SAE J 126 & $\begin{array}{l}\text { Selección y Especificación de Lámina y Cinta de Acero } \\
\text { Laminado en Frío y en Caliente }\end{array}$ \\
\hline SAE J 402 & $\begin{array}{l}\text { Nuevo Sistema de Designación de Acero para Acero Forjado o } \\
\text { Laminado }\end{array}$ \\
\hline SAE J 911 & $\begin{array}{l}\text { Medición de Conteo Máximo y Rugosidad de Superficie de } \\
\text { Lámina de Acero Laminado en Frío }\end{array}$ \\
\hline SAE J 1392 & $\begin{array}{l}\text { Lámina y Cinta de Acero, de Alta Resistencia, Laminado en } \\
\text { Frío, Lámina con Laminado en Frío, y Lámina con } \\
\text { Revestimiento }\end{array}$ \\
\hline SAE J 1442 & Barras de Acero, Laminado en Caliente y de Alta Resistencia \\
\hline SAE J 2281 & $\begin{array}{l}\text { Selección y Especificación de Productos de Barras de Acero } \\
\text { Laminado en Caliente }\end{array}$ \\
\hline
\end{tabular}




\section{ENTIDADES DE CERTIFICACIÓN Y PRUEBAS}

\section{Pruebas}

Numerosos laboratorios hacen pruebas en acero y demás materiales para normas reconocidas de la industria; algunos pueden estar acreditados. Se puede encontrar una lista de los laboratorios de pruebas acreditados en A2LA Listado de Laboratorios Acreditados por Prueba. Listados de otros laboratorios de pruebas pueden encontrarse en ASTM Directorio de Laboratorios de Pruebas. Buscar la palabra clave "steel" (acero) o una norma ASTM específica.

\section{Certificación}

No se identificaron programas de certificación para acero laminado. Todas las importaciones de los Estados Unidos de productos básicos de acería están sujetos a los requerimientos de licencia de importación según el 19 CFR 360 Sistema para el Monitoreo y Análisis para la Importación de Acero a través del sistema para el Monitoreo y Análisis para la Importación de Acero (SIMA) basado en la Internet.

\section{AGENCIAS DE GobIERNo RELEVANTES DE ESTADOS UNIDOS}

\section{Comisión Federal de Comercio}

600 Pennsylvania Avenue, NW

Washington, DC 20580

Teléfono: +1.202 .326 .2222$

Lista de Contactos

\section{Oficina de Aduanas y Protección Fronteriza (CBP) de los Estados Unidos} 1300 Pennsylvania Avenue, NW

Washington, D.C. 20229 USA

Teléfono: +1.703 .526 .4200$

Lista de Contactos

INDUSTRIA DEL ACERO Y DATOS DE MERCADO

\section{Asociaciones de Comercio de la Industria}

Instituto Americano del Hierro y el Acero (AISI)

25 Massachusetts Avenue, NW Suite 800

Washington, DC 20001

Teléfono: +1.202 .452 .7100$ 
El AISI está compuesto por 25 empresas integrantes, incluyendo fabricantes siderúrgicos de hornos integrados y eléctricos, y 124 miembros asociados y afiliados que corresponden a proveedores o clientes de la industria del acero. Las empresas integrantes del AISI representan más de tres cuartas partes de la capacidad de producción de acero de los Estados Unidos y Norteamérica.

\section{Asociación de Fabricantes de Acero}

150 Connecticut Avenue, NW Suite 715

Washington, D.C. 20036

Teléfono: +1.202 .296 .1515$

La asociación de fabricantes de acero consta de 36 productores de acero norteamericanos que representan más del $75 \%$ de la producción de acero local.

\section{Datos de Mercado sobre el Acero Laminado}

Instituto Americano del Hierro y el Acero

Perfil 2013 del Instituto Americano del Hierro y el Acero 
El Centro de Información sobre Estándares (SIC) del NIST realiza los esfuerzos necesarios para proporcionar información exacta y completa. Varios datos como nombres, números de teléfono, vínculos a las páginas web, etc., pueden cambiar antes de la actualización. Agradecemos sus sugerencias sobre cómo mejorar la presente guía y corregir los errores. El SIC brinda esta información "TAL CUAL APARECE." EI NIST y el SIC NO DAN NINGUNA GARANTÍA DE NINGÚN TIPO, incluyendo NINGUNA GARANTÍA DE COMERCIABILIDAD O ADECUACIÓN PARA NINGÚN PROPÓSITO ESPECÍFICO. EI NIST no garantiza ni asume responsabilidad alguna por la exactitud, precisión, integridad o confiabilidad de la información. Como condición para el uso de la guía de información, usted explícitamente libera al NIST/SIC de cualquier responsabilidad por cualquier daño o perjuicio de cualquier tipo causado que pueda resultar por los errores u omisiones en la presente Guía o cualquier otro dato. Algunos de los documentos que se referencian apuntan a información creada y revisada por otras organizaciones. El SIC no controla y no puede garantizar la relevancia, periodicidad o exactitud de dichos materiales.

Noviembre de 2017

Preparado por el Centro de Información sobre Estándares (SIC)

Coordinación de Normas (SCO)

Instituto Nacional de Normas y Tecnología (NIST)

standardsinfo@nist.gov

http://www.standards.gov 